\title{
Bingo from SciTouch LLC: chemistry cartridge for Oracle database
}

\author{
Dmitry Pavlov", Mikhail Rybalkin, Boris Karulin \\ From 5th German Conference on Cheminformatics: 23. CIC-Workshop \\ Goslar, Germany. 8-10 November 2009
}

Bingo is a data cartridge for Oracle database that provides the industry's next-generation, fast, scalable, and efficient storage and searching solution for chemical information.

Bingo seamlessly integrates the chemistry into Oracle databases. Its extensible indexing is designed to enable scientists to store, index, and search chemical moieties alongside numbers and text within one underlying relational database server.

For molecule structure searching, Bingo supports 2D and $3 \mathrm{D}$ exact and substructure searches, as well as similarity, tautomer, Markush, formula, molecular weight, and flexmatch searches. For reaction searches, Bingo supports reaction substructure search (RSS) with optional automatic generation of atom-to-atom mapping. All of these techniques are available through extensions to the SQL and PL/SQL syntax.

Bingo also has features not present in other cartridges, for example, advanced tautomer search, resonance substructure search, and fast updating of the index when adding new structures.

The presentation itself you can download from our site: http://opensource.scitouch.net/downloads/bingo-cic. pdf

Published: 4 May 2010

doi:10.1186/1758-2946-2-S1-F1

Cite this article as: Pavlov et al:: Bingo from SciTouch LLC: chemistry

cartridge for Oracle database. Journal of Cheminformatics 2010

2(Suppl 1):F1.

* Correspondence: dpavlov@scitouch.net

SciTouch LLC, Budapeshtskaya st. 23-2-82, RUS-192212 St. Petersburg, Russia

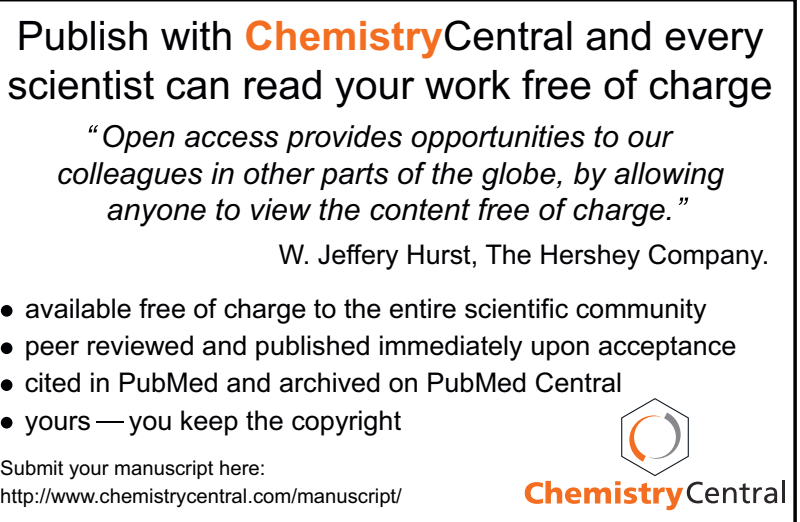

